\title{
Application of Fuzzy Variable Structure Control in DC Source of Plasma Arc Welding
}

\author{
Xin-wei LI \\ Shangdong Transport Vocational College \\ Weifang,China \\ e-mail: lixinwei1511@163.com
}

\author{
Xiang-min CHEN \\ Shangdong Transport Vocational College \\ Weifang,China \\ e-mail: 254405685@qq.com
}

\begin{abstract}
A new control system of DC source of plasma arc welding was designed according to the shortcomings of timevarying and nonlinearity in the design process of DC source of plasma arc welding and traditional control algorithms could not be overcome these disadvantage. Main circuit and control circuit were included in this system. Fuzzy variable structure control was used to control the PWM module circuit combining the fuzzy control and traditional PID control algorithm. The simulation results showed that the system has high dynamic performance and steady performance and has some value of popularization and application.
\end{abstract}

Keywords- DC source of plasma arc welding; fuzzy variable structure control; style; small-signal modeling; simulation

\section{INTRODUCTION}

The plasma arc is a kind of arcs with high temperature, high ionization degreen high energy using a plasma gun compressing the cathode (such as tungsten) and the anode ${ }^{[1-}$ 2]. Plasma arc welding has the characteristics of heat input energy concentrating, large ratio of depth to width, high welding efficiency, and small deformation after welding. Plasma arc power supply should have a steep drop or the rappelling characteristics, and the no-load voltage of the power supply should be more than 2.5 times of the voltage of plasma arc. The change in current in the welding process, the flow of plasma gas and torch operation control need to be precisely adjusted in real time which put forward higher requirements for the performance and accuracy of the welding process controller.

Centralized control system based on PLC is generally used in welding automation control system produced by domestic welding machine manufacturers, but it is difficult to realize complex welding process control. Currently, universities and research institutions in China have begun research of welding automation control system based on field bus technology, but the diversity and standard field bus unified make the field bus technology difficult to get a greater area of promotion and application. Digital plasma DC welding power was designed based on the ARM Cortex-M3 core chip from the point of reducing the cost and difficulty of development in order to obtain better accuracy and response speed of control, and using fuzzy variable structure control algorithm was used to control the arc welding power source in order to overcome the impact of the uncertainties to power performance during operation.

\section{Overall System DESIGN}

The main power source circuit shown in Figure 1, is the hardware carrier of completing energy transmission. Current waveform is conversed by controlling the turn-on and turnoff of the main circuit switching tube to obtain a desired welding current and voltage waveforms. The form of energy converter is AC-DC-AC-DC. Inverter circuit using a fullbridge circuit has the advantage of large output power. Fullbridge circuit can achieve maximum power in all isolated switching circuit using same voltage and current capacity of the switching device.

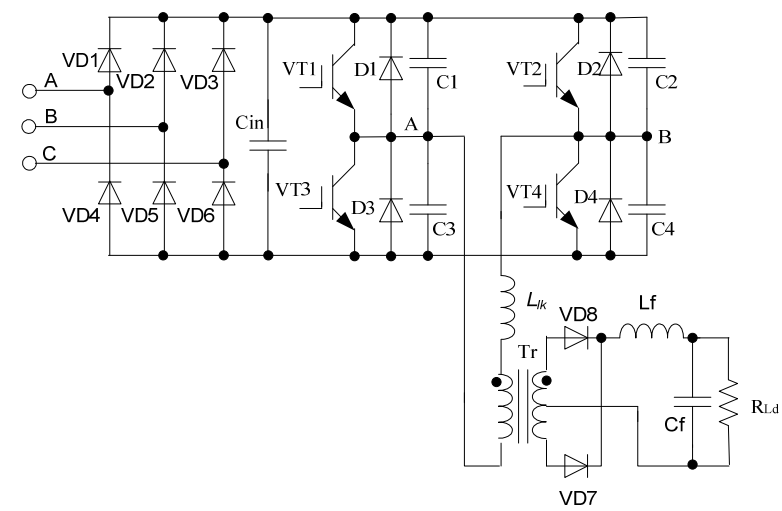

Figure 1. Main circuit

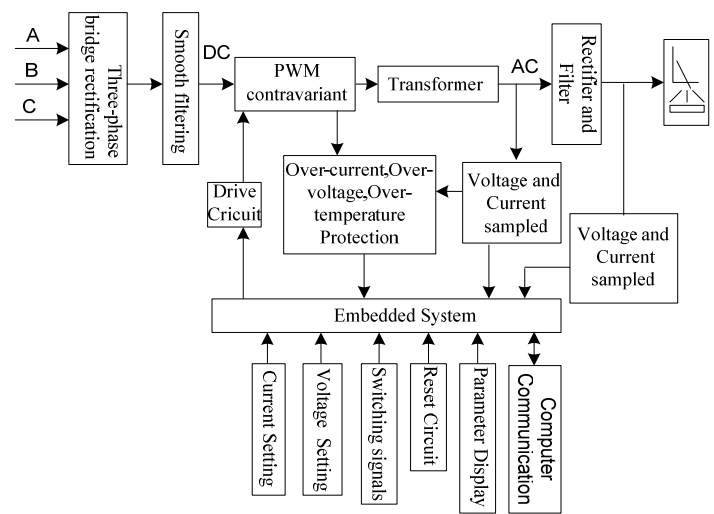

Figure 2. Structure diagram of switching power supply

The entire system shown in Figure 2 includes the main circuit, the control circuit, the operating system and the software program ${ }^{[3]}$. MCU collects field data such as welding current and arc voltage, and makes Data process 
and compute to output a control signal. This signals control the PWM interface in real time to output PWM pulse signal of proper width. The pulse signals drive the IGBT gate after driver amplifier to control the real-time adjustment of the output current and voltage of the power supply.

\section{CONTROL Algorithm Design}

\section{A. Small-signal Modeling}

The structure of the switching power supply can be shown as Figure 3. Regulator include control and correction links ${ }^{[4]}$, and it is assumed that the transfer function is $G_{1}(s)$. Time proportional control and pulse forming part of the linear model can be represented as a scaling factor, denoting as $K_{1}$. High-frequency transformer, rectifier and filter links can be instead by a scaling factor, together with the inverter main circuit as $G_{2}(s)$. When the output is voltage or current value, the feedback circuit is generally proportional component. When the output is indirect amount such as frequency, etc., it may be one order inertial link. Transfer function is $K_{3}$ in this paper. Filtering link is an LC low-pass filter, whose transfer function is $\frac{1}{s^{2} L C+1}$. As shown in

Figure 4 its closed-loop transfer function is:

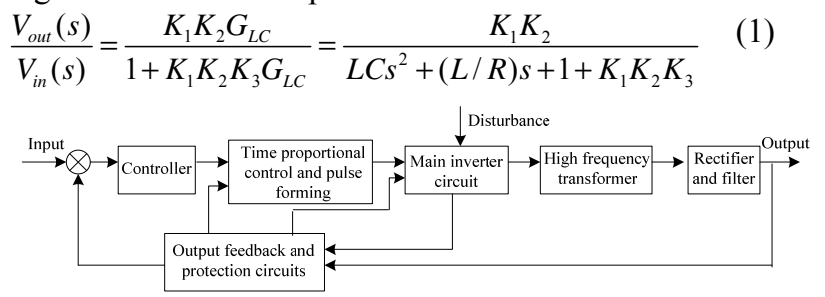

Figure 3. Structure diagram of switching power supply

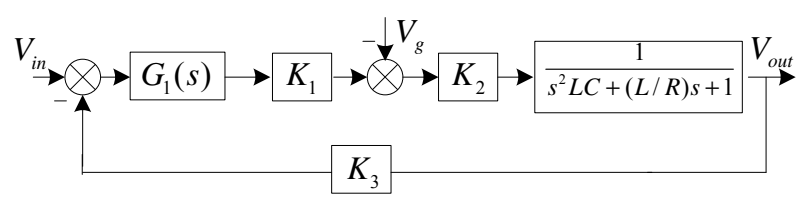

Figure 4. Whole frame diagram of control system

We can see from the step response curve as shown in Figure 5.There are principle errors in the system under no control and the system has a bigger overshoot and a longer mediation time of $1 \mathrm{~ms}$. Therefore, a certain control algorithm must be used to make the system stable operate.

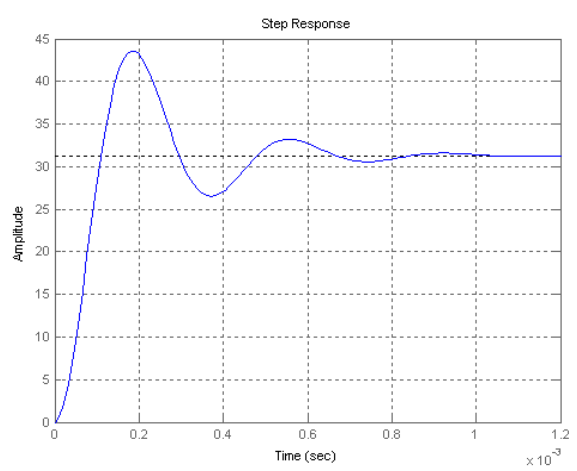

Figure 5. Step response of system under no controller

\section{B. Fuzzy Variable Structure Control Algorithm}

Mathematical model of welding power in the small signal range can be established, and application of PID control algorithm can be applied for more accurate control. But plasma spray welding power supply is a strongly nonlinear, time-varying system, and much disturbance may happen frequently such as large-signal input, voltage or load change, change in the operating point of the system, so the smallsignal model is not longer applicable. Plasma spray power is difficult to establish a precise mathematical model in the entire operating range, so the application of the PID control algorithm is restricted. So fuzzy control after all, is a program worth considering ${ }^{[5-6]}$.

Fuzzy control is based on organization and control the decision-making table of the manual control rules, and the table determines the amount size. Knowledge representation in control, fuzzy rules and synthetic reasoning are from the mature experience, expert knowledge or skilled operator. They can effectively improve the nonlinear dynamic characteristics of the region, and can be able to overcome the impact of the non-linear factors, apply the non-linear system. But its simple fuzzy processing for the information will cause the system accuracy too low. The quantization levels must be increased to improve the control accuracy. As a result the number of computing is greatly increased and reduces the speed of fuzzy decision. For a twodimensional fuzzy controller the input of the controller have only the amount of deviation and its change rate of the amount charged. It is actually equal to a PD control with variable parameters. Because there is no consideration of the integral role, it is difficult to eliminate the presence of the steady-state error.

Fuzzy variable structure control algorithm as shown in Figure 6 was used according to the advantages and disadvantages of these two kinds of algorithms. Fuzzy control and PID control are treated differently depending on the situation in the control process. This method can not only maintain the advantage simple control principle and good robustness of conventional PID control system, but also play the adaptability and flexibility of fuzzy control. 


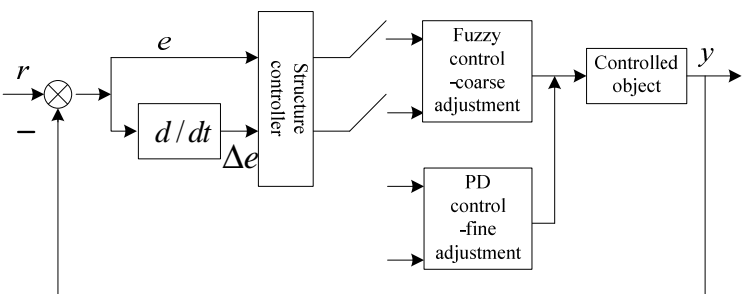

Figure 6. Structure diagram of fuzzy variable structure control

The standard universe of input $e, \triangle e$ and output $\triangle u$ are $E=[-3,+3], \quad E C=[-3,+3], \Delta U=[-3,+3]$. Five fuzzy variables are negative large (NB), negative small (NS), zero ( $\mathrm{ZO}$ ), positive small (PS), positive large ( $\mathrm{PB}$ ) . Membership functions are triangular membership function as shown in Figure 7.
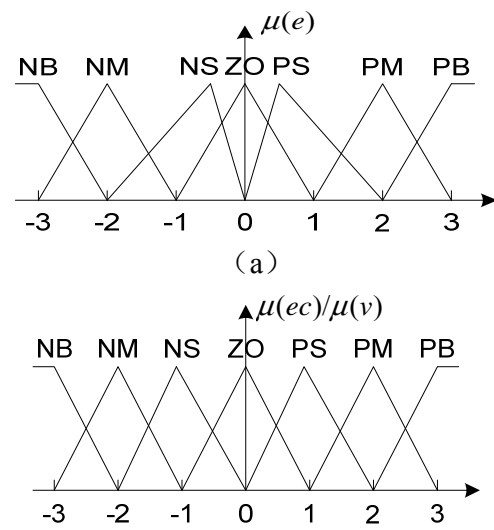

(b)

Figure 7. Membership functions: (a) $E$ (b) $E C$ and $V$

The controller according to the size of the error is divided into two work intervals "coarse adjustment"and "fine adjustment". When the system output and the set value have a larger deviation, the object of control action is to reduce the error as soon as possible, so the interval of the work in the "coarse adjustment". The fuzzy control algorithm was used during "coarse adjustment", and the controller structure is shown in Figure 8.Its fuzzy subset is $e, \Delta e=\{N L, N S, Z O, P S, P L\}$, elements of subset represent negative large, negative small, zero, positive small, positive large. Membership function of deviation and deviation variation rate is shown in Table 1 . When the system is around the set value, it comes into the "fine adjustment" range. Control purposes at this time are making the output error as small as possible. That is to say it eliminates the steady state error of the system. The analysis shows that in the welding process the current range does not change too much. Therefore, the controller is actually only a few time working in the "coarse" period, and more time working in the "fine-tuning" period.

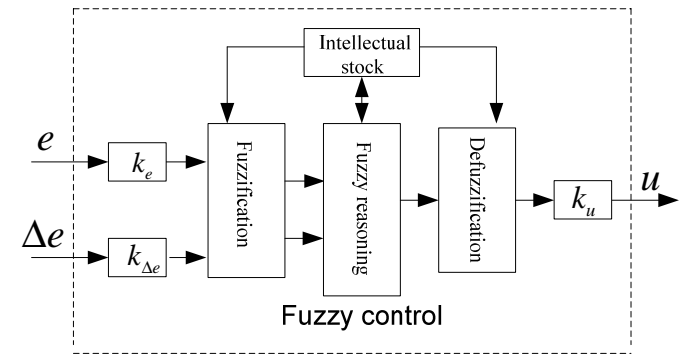

Figure 8. Structure diagram of fuzzy controller

\begin{tabular}{l|l|l|l|l|l}
\multicolumn{7}{c|}{ Table 1. Rule list of fuzzy controller } \\
\hline NL & NL & NS & ZO & PS & PL \\
\hline NS & PL & PS & A & A & NL \\
\hline ZO & PL & A & ZO & A & NL \\
\hline PS & PL & A & X & NS & NL \\
\hline PL & PL & NS & NS & NL & NL \\
\hline
\end{tabular}

The working process of fuzzy controller is as follows. Firstly, the multiplication of $e, \triangle e$ and quantization factor was transformed into the $E$ the $E C$ universe $[-3,3]$. The amount of control $V$ was obtained by the method of defuzzification though weighted average method and fuzzy control rule table (Table 1); Finally, the control amount $V$ multiplied by the scale factor to obtain adjustable coefficient $v$.

$A$ in the control rule table of the system presents entering the "fine adjustment" zone. The "fine adjustment" stage uses PD algorithm. Output quantity:

$$
u=\frac{x e+y \Delta e}{x e^{\prime}+y \Delta e^{\prime}}
$$

In the formula, $x, y$-Weights, the weights of $e$ and $\Delta e$ $e^{\prime}$ - the maximum deviation of fine adjustment;

$\Delta e^{\prime}$ - the deviation variation rate of fine adjustment.

\section{ANALYSIS AND AIMULATION RESUltS}

Simulation models were built by Simulink blocks in Matlab including 6 modules of the rectifier, inverter, transformer, high-voltage rectifier, controller and PWM pulse generator. In order to make switching power supply work efficiently at a higher frequency with low power consumption, safe operation and the switching losses greatly be reduced, the soft-switch technology were used in the PWM modulation circuit. Figure 9 are a driving waveform of the switching tube VT4 and a voltage waveform between collector regions and emitter regions. The switch voltage was zero in the time of tube turn-on and turn-off time, and realized zero-voltage switching control. 

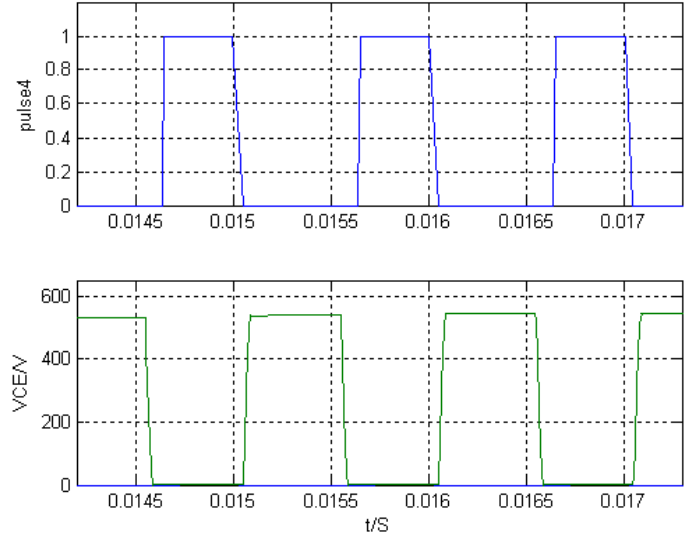

Figure 9. Waveform of driving and switch tube voltage

Power dynamic characteristic is one of the important indicators to measure the performance of the power supply. Two aspects of the simulation were carried out because the power supply is a constant current source in order to verify the system's dynamic characteristics. The first one is the current response under setting signal singularity, and the second one is the simulation under the suddenly change of the loads. Figure 10 is the current response under setting signal singularity when the setting current is $70 \mathrm{~A}$ and the initial load is $0.2 \Omega$. The current transition time is about $30 \mu \mathrm{s}$ under fuzzy variable structure control. And fuzzy variable structure control can quickly overcome when increase a $2 \mathrm{~A}$ current disturbance in the time of $200 \mu \mathrm{s}$. Thus, the system has a better dynamic performance indicators.

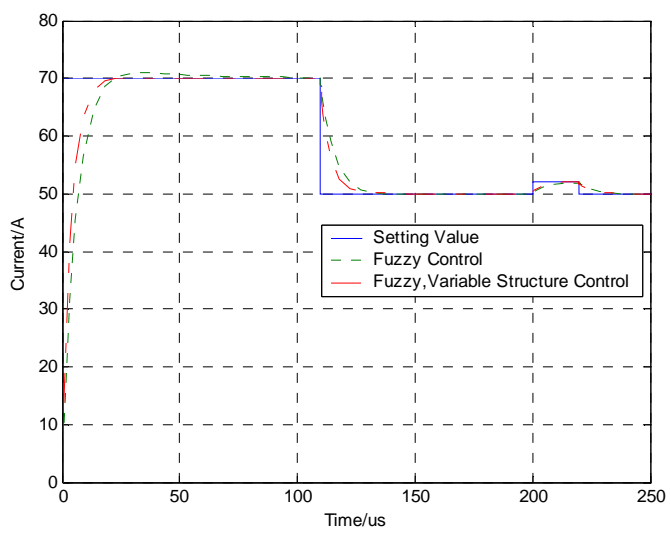

Figure 10. Waveforms of current under control

\section{RESUlTS}

The welding power can not achieve good control effect using the traditional control mode because of its disadvantages of time-varying, nonlinear. The embedded system as the core hardware and fuzzy variable structure control algorithm were presented in this paper. And the dynamic and steady-state performance of DC plasma arc welding power has been greatly improved both the reliability and the development efficiency. Finally the feasibility of the design is verified by simulation.

\section{REFERENCES}

[1] WU Lei,LI Peng-cheng, YUAN Bi-jin. Research on Application of a Combined Induction Heating Power Modulation Based on Fuzzy Logic Control in Power Electronics,2010,44(7):53-55. Vol.44 No.7 Jan. 2010,pp. 53-55.

[2] Yanfeng Chen, Shuisheng Qiu, Yanzheng Wu. Extension of characteristic equation method to stability analysis of equilibrium points for closed-loop PWM power switching converters[J]. Communications in Nonlinear Science and Numerical Simulation, Vol.4 No.4, 1999,pp. 276-280.

[3] ZHANG Dong,WANG Zhen-min,LI Jin,WANG Zhi-qiang. Development of digital tube-plate welding power source based on ARM Cortex - M3[J]. Welding Technology,Vol.39 No.1 Jan. 2010,pp.41-44.

[4] LU Zheng-yang,YAN Huo-tong,CHEN Shu-jun,ZHANG Jun,LIANG Shuai. "Contorl system of the variable polarity plasma arc welding based on fieldbu" in Electric Welding Machine,Vol.41 No.1. 2011,pp.15-20.

[5] Kemal Kilic, Beth A Sproule, I Burhan Türksen, Claudio A Naranjo. A fuzzy system modeling algorithm for data analysis and approximate reasoning in Robotics and Autonomous Systems, Vol.49 No.3. 2004,pp.173-180.

[6] Ning Li, Shao-Yuan Li, Yu-Geng Xi. Multi-model predictive control based on the Takagi-Sugeno fuzzy models: a case study[J]. Information Sciences, 2004, 165(3): 247-263 